\title{
Welchen Patientennutzen bringt die Handchirurgie?
}

\section{Michaël Papaloïzos}

Ehemaliger Präsident und Vorstandsmitglied der Schweizerischen Gesellschaft für Handchirurgie

Dank der Handchirurgie erhöht sich nicht nur die Lebensqualität der Patienten, weil sie Hand, Handgelenk und Arm wieder bestmöglich gebrauchen können. Da ihnen dadurch ihre beruflichen wie häuslichen Tätigkeiten wieder möglich werden, leisten sie auch einen direkten wie indirekten positiven Beitrag zur Volkswirtschaft.

\section{Ein Fachgebiet, das der gesellschaftlichen und wirtschaftlichen Bedeutung der Hand gerecht wird}

Die Schweizerische Gesellschaft für Handchirurgie wurde 1966 in Lausanne gegründet. Professor Claude Verdan, die treibende Kraft hinter dieser Gründung, hatte wie andere Akteure in Europa und den USA zwei wichtige Aspekte erkannt: die wirtschaftliche Bedeutung von Hand und Handgelenk - deren Verletzungen ein Viertel aller Notfallbehandlungen ausmachen und die Notwendigkeit eines spezifischen Ansatzes, da diese Verletzungen häufig unzureichend behandelt und zahlreiche Patienten arbeitsunfähig wurden, was zulasten der gesunden Beitragszahler ging [1].

Fünfzig Jahre später hat die Handchirurgie den Status einer eigenständigen Disziplin erreicht. Für die Anerkennung und die ständige Weiterentwicklung dieser Spezialisierung ist in erster Linie der Mehrwert verantwortlich, den sie sowohl den einzelnen Patienten als auch der Gesellschaft insgesamt gebracht hat.

\section{Finger gerettet, (Arbeits-)Leben gerettet!}

Das Verdienst Verdans liegt insbesondere darin, die Chirurgie der Beugesehnen revolutioniert zu haben. Seitdem hat sich diese Chirurgie ständig weiterentwickelt und sorgt nun dafür, dass Finger, deren Funktionstüchtigkeit früher ein für alle Mal verloren gewesen wäre, wieder beweglich werden - bei Schreinern ebenso wie bei Musikern.

Heute kann man sich oft nicht mehr vorstellen, was für ein Schicksalsschlag eine solche Verletzung früher für die Betroffenen bedeutete. Verdan empfing einmal einen jungen Schreinerlehrling, der ihn - im vollen Bewusstsein dessen, was für ihn auf dem Spiel stand - an- flehte: «Meine Hände sind alles, was ich habe. Bitte retten Sie sie mir!» Seitdem haben sich die Techniken zur Reparatur wie auch zur Rehabilitation der Beugesehnen enorm weiterentwickelt und verfeinert. Heutzutage müsste sich dieser Patient keine solchen Sorgen mehr um die Funktionstüchtigkeit seiner Hand und um seine Arbeitsfähigkeit machen.

Es ist sogar noch viel mehr möglich, wie ein anderes Beispiel zeigt: Eine junge Konzertpianistin erlitt bei einem einfachen Sturz eine Fraktur des ersten Glieds ihres kleinen Fingers, der danach verformt und nicht mehr benutzbar war. Noch vor zwei oder drei Jahrzehnten wäre eine solche Fraktur kaum therapierbar gewesen. Sehr häufig blieben die Finger in solchen Fällen dauerhaft steif und unbrauchbar. Dank neuartiger Implantate und Techniken konnte diese Patientin operiert und die Fraktur stabilisiert werden, so dass sich der Finger rasch mobilisieren liess und die einstmals fast unvermeidlichen Versteifungen ausblieben. Drei Monate später absolvierte die Musikerin wieder Orchester- und Soloauftritte. Die mehreren tausend Franken, welche dieser Eingriff und die Reha gekostet haben, waren mit Sicherheit gut investiertes Geld, da sonst eine ganze Karriere als Konzertmusikerin vor dem Ende gestanden hätte.

\section{Ein gerettetes Handgelenk $=$ ein Fall von Berufsunfähigkeit weniger}

Die häufigste Fraktur ist die des Handgelenks. Sie betrifft insbesondere ältere Frauen - eine Entwicklung, die sich noch verstärken dürfte. Noch Ende der achtziger Jahre behandelte man solche Frakturen in der Regel durch Reponieren und Eingipsen oder mit Hilfe einer externen Fixierung. Dies war nicht nur lästig, 
sondern häufig auch erfolglos und Ursache schwerwiegender Spätfolgen in Form steifer und schmerzender Handgelenke. Die Meinungen waren geteilt, die Richtlinien vage und unzureichend begründet - kurz, man

\section{Neue Behandlungen verschaffen den Patienten Unabhängigkeit, so dass sie zu Hause bleiben oder zumindest rasch wieder zurückkehren können.}

fischte im Trüben, und Leidtragende des niedrigen Wissensstands der damaligen Zeit waren junge wie ältere Patienten mit ihren Frakturen. Die zu Beginn der 2000er Jahre aufgekommenen Fixierungsplatten haben alle Beteiligten überzeugt und sich rasch als eindeutiger Standard in der Behandlung der meisten Handgelenkfrakturen durchgesetzt. Diese Platten verschaffen dem Handgelenk in kurzer Zeit wieder Mobilität und Funktion - und den Patienten Unabhängigkeit, so dass sie zu Hause bleiben oder zumindest rasch wieder zurückkehren können. Die Kosten einer solchen Platte (ca. 1200 Franken) und des Eingriffs (ca. 1500 Franken laut TARMED) stehen in keinem Vergleich zu den direkten und indirekten Kosten einer dauerhaften Behinderung junger wie älterer Patienten.

Hinzu kommen noch andere Fortschritte: Mikrochirurgie und Reimplantationen, Endoprothesen für Handund Fingergelenke, minimalinvasive und endoskopische Chirurgie usw. - eine vollständige Liste wäre lang. Noch mehr als die neuen Instrumente zählt allerdings ihr kunstfertiger Einsatz - der eine spezialisierte Ausbildung erfordert.

\section{Ein zusätzliches Fachgebiet, das den Patienten bestmögliche Qualität gewähr- leistet}

Ein Spezialist für Handchirurgie gewährleistet, dass die Therapie nicht nur dem Leiden, sondern dem ganzen Patienten mit seinen individuellen Umständen optimal entspricht - sei es in Notfall- oder in Elektivsituationen. Eine Arthrose am Daumensatz ist bei einem 35-jährigen Mann, bei dem sie auf eine Fraktur zurückzuführen ist, anders zu behandeln als eine degenerative Arthrose bei einer Frau von 65 Jahren. Zwar stellen beide Krankheitsbilder ein Handicap dar, aber eben doch in unterschiedlichen Kontexten, die bei der Wahl der Therapie berücksichtigt werden müssen.

Es ist belegt, dass eine unsachgemässe Behandlung durch nicht spezialisiertes Personal klar schlechtere Ergebnisse liefert und letzten Endes höhere Kosten verursacht [2]. Wird frühzeitig ein Spezialist aufgesucht, ist zumeist eine bessere Therapie gewährleistet [3]. Ein gutes Beispiel ist die häufig zu beobachtende Tendovaginitis de Quervain, also eine Entzündung der Sehnenscheide am Handgelenk. Immer wieder sehen Handchirurgen solche Patienten erstmals, nachdem diese dutzende Male in der Physiotherapie waren, (mehr oder weniger geeignete) Schienen erhalten haben, teure Untersuchungen über sich haben ergehen lassen, arbeitsunfähig geschrieben und seit Wochen quasi depressiv sind, "weil niemand sagen kann, was ich eigentlich habe». Und das, obwohl eine klinische Untersuchung in den meisten Fällen zur Diagnose ausreichen und die lokale Injektion eines Entzündungshemmers oder ein einfacher, aber präziser ambulanter chirurgischer Eingriff eine Heilung in drei bis vier Wochen gewährleisten würde - zu niedrigsten Kosten.

Die Handchirurgie stellt die funktionellen Fähigkeiten und die Autonomie der Patienten wieder her und vermeidet die Kosten einer dauerhaften Behinderung.

In der Handchirurgie hat der Übergang von der stationären zur ambulanten Behandlung in der Regel schon stattgefunden: 80 bis $85 \%$ der ärztlichen Eingriffe sind hier ambulant. Diese Quote ist in Spitälern niedriger die einen höheren Anteil schwierigerer Pathologien und Patienten tragen. Mit einer einheitlichen Finanzierung ambulanter und stationärer Leistungen und angemessenen Tarifen könnte diese Quote zweifellos noch höher sein - was allen Beteiligten zugute käme.

\section{Zusammenfassung}

Ob infolge eines Unfalls, ob durch eine scheinbar banale Verstauchung oder eine ernsthafte Erkrankung, ob im Falle von Arthrose oder chronischer Schmerzen, ob bei alten oder jungen Patienten - Handchirurgen gewährleisten die bestmögliche Erst- und Weiterbehandlung. Die Diagnosen und Therapien dieser Spezialisten lindern das Leid und sorgen für eine höhere Lebensqualität. Darüber hinaus stellt die Handchirurgie die Arbeitsfähigkeit und Autonomie der Patientinnen und Patienten wieder her und vermeidet dadurch die direkten und indirekten Kosten einer dauerhaften Behinderung.

\section{Literatur}

1 Dias JJ, Garcia-Elias M. Hand injury costs. Injury, Int J Care Injured. 2006;37:1071-7.

2 Kenesi C, Masmejean E. Les urgences main en France. Un important défi de santé publique. Bull Acad Natl Med. 2004;188:793-800.

3 Raimbeau G. Coûts des urgences mains. Chir Main. 2003;22:258-63. 